Compared with systemic lupus erythematosus (SLE) non active LN patients, the active LN group showed lower basophil counts $(p=0.002)$. Moreover, Significant differences were found in circulating basophil counts among LN pathology classification groups $(p<0.05)$. Groups of classes III, IV were more likely to have lower circulating basophil counts when compared with the other classes $(p=0.047)$.

Conclusion According to these results circulating basophil counts might be an interesting marker for renal activity of LN.

\section{PS5:108 SEMAPHORIN3A: A BENEFICIAL THERAPY FOR LUPUS NEPHRITIS}

E Toubi, Z Vadasz. Bnai-Zion Medical Centre, The Technion Faculty of Medicine, Haifa, Israel

\subsection{6/lupus-2018-abstract.152}

Background The immune regulatory properties of semaphorin3A (sema3A) (both innate and adaptive) are well established in many in-vitro studies. When sema3A was incubated with active B cells from SLE patients, it could efficiently reduce the expression of TLR-9 in correlation with a significant reduction of relevant autoantibodies. The injection of sema3A to a mice model of rheumatoid arthritis was proven to be highly beneficial, both in attenuating clinical symptoms and in decreasing inflammatory mechanisms.

Objectives This study was designed in order to assess possible therapeutic benefits following the injection of sema3 A to NZB/W mice. Results The injection of sema3A to young mice (at week 12) before disease onset, delayed the appearance of proteinuria. Here, the median time to severe proteinuria was 110 days, 95\% CI: 88 to 131. However, in mice in which empty vector was injected, median time to severe proteinuria was 63 days, 95\% CI: 0 to 139). Sema3A treatment, reduced significantly renal damage, namely, it prevented the development of immune deposits in the glomeruli. When sema3A was injected at the onset of proteinuria, aiming to treat rather than to prevent disease in these mice, survival was significantly increased and the deterioration of proteinuria was significantly delayed. Conclusion Semaphorin3A is highly beneficial in reducing lupus nephritis in $\mathrm{NZB} / \mathrm{w}$ mice. It delays the appearance and deterioration of proteinuria, and increases survival rates in these mice. Further studies will establish the idea of applying sema3 A in the treatment of lupus nephritis.

\section{Poster session 6: Management and recommendations, diagnostic and classification criteria}

\section{PS6:109 ASSOCIATION OF MENTAL HEALTH RELATED QUALITY OF LIFE (HRQOL) AND SUBSEQUENT PHYSICAL FUNCTIONING IN SYSTEMIC LUPUS ERYTHEMATOSUS: ANALYSIS OF DATA FROM THE GERMAN LULA COHORT $2002-2013$}

${ }^{1} R$ Brinks, ${ }^{1} G$ Chehab, ${ }^{1} R$ Fischer-Betz, ${ }^{1}$ Jichter, ${ }^{1} R$ Willers, ${ }^{2} B$ Winkler-Rohlfing, ${ }^{1} \mathrm{M}$ Schneider. 'University Hospital Duesseldorf, Department and Hiller Research Unit for Rheumatology, Düsseldorf, Germany; ${ }^{2}$ Self help group for Lupus Erythematodes, Wuppertal, Germany

\subsection{6/lupus-2018-abstract.153}

Introduction Mental symptoms are a major problem in patients with systemic lupus erythematosus (SLE). From geriatric patients it is well-known that prevention of mental disorders leads to maintenance of physical functioning (PF).

Methods Data from the German Lupus-Langzeitstudie (LuLa) open cohort was used to explore the effect of self-reported mental HRQoL on PF. The ongoing LuLa study comprises patients from the German SLE self-help group. The current analysis uses data from 2002 to 2013. We looked for associations between the PF score (outcome) and the mental component summary (MCS) score (exposure) of the SF-12 questionnaire in one to four years preceding the outcome. Statistical analysis was accomplished by a linear mixed regression model (LMM) adjusting for the potential confounders age, sex, disease duration and disease activity as surveyed by the Systemic Lupus Activity Questionnaire score (SLAQ).

Results 1511 patients $(93.3 \%$ women) provided data with a total of 7841 person-years of observation. Median age at study entry was 46 (interquartile range: 37 to 57). Table 1 shows the regression coefficients of MCS (and 95\% confidence intervals, CI) estimated by the LMM without and with adjusting for age, sex, disease duration and SLAQ.

Conclusions We found evidence that self-reported mental HRQoL in patients with SLE is associated with PF in the subsequent years. The data indicate a 'window of opportunity' for treating mental symptoms of up to three years for maintenance of PF.

Abstract PS6:109 Table 1 Association between physical functioning (outcome) and mental health related quality of life (MCS) one to four years before the outcome

\begin{tabular}{|l|r|r|r|r|}
\hline \multirow{2}{*}{$\begin{array}{l}\text { Exposure: Mental component } \\
\text { summary score (MCS) }\end{array}$} & \multicolumn{2}{|c|}{ Crude estimates } & \multicolumn{2}{c|}{ Adjusted estimates } \\
\cline { 2 - 5 } & Estimate, 95\% CI & p-value & Estimate, 95\% CI & p-value \\
\hline 1 year ahead & $0.12,(0.07,0.17)$ & $<0.001$ & $0.09,(0.04,0.13)$ & $<0.001$ \\
2 years ahead & $0.12,(0.08,0.17)$ & $<0.001$ & $0.09,(0.04,0.13)$ & $<0.001$ \\
3 years ahead & $0.07,(0.02,0.12)$ & 0.009 & $0.06,(0.01,0.11)$ & 0.016 \\
4 years ahead & $0.02,(-0.04,0.07)$ & 0.564 & $0.02,(-0.03,0.08)$ & 0.395 \\
\hline
\end{tabular}

